\title{
Estimation of Crystal Sample Structure by Means of Fast Electron Radiation
}

\author{
D. A. Baklanov, I. E. Vnukov, Yu. V. Zhandarmov, and R. A. Shatokhin \\ Belgorod State University, Belgorod, Russia
}

Received June 8, 2009

\begin{abstract}
The dependence of the characteristics of X-ray radiation occurring under fast electron transmission through a crystal on a sample structure has been analyzed. A method to estimate the size of the crystal microblocks by means of the yield ratio of the parametric X-ray radiation and diffracted bremsstrahlung and transition radiation for different observation angles has been suggested. The possibility of estimating the dimension of the microblocks using the ratio of the intensities of these emission mechanisms for the same observation angle and several reflection orders has been discussed.
\end{abstract}

\section{INTRODUCTION}

Ordering of the environment atoms leads to the occurrence of orientation and interference effects in the yield of secondary processes arising at the passage of fast charged particles through it. In particular, these are processes of dispersion, yield of nuclear reactions, generation of radiation, etc. This connection allows one to analyze target structures using the results of measurements. For example, using the backscattering yield of channeling ions, one can make a judgment about the arrangement of impurities in the crystal lattice, and using the radiation spectra at channeling of fast electrons, one can specify the form of the potential and the density of electrons [1].

Analyzing the quality of the structure of crystal samples, i.e., the presence of mosaic blocks in the sample, their distribution on the disorientation angle with respect to the main direction and on the size, and on the characteristics of the X-ray radiation generated at the passage of fast electrons through them, is a similar problem. The advantages of this approach are a high penetrating ability of X-ray radiation and visualization of interpretation. Recording and analysis of the characteristics of the radiation that is irradiated at the large angles to the direction of the incident particles on the sample that considerably reduces the contribution of the bremsstrahlung radiation that is insensitive to the structure of the sample appear to be most convenient.

Two kinds of radiation meet this requirement: parametric X-ray radiation (PXR) [2, 3], which can be considered as the coherent dispersion of the intrinsic electromagnetic field of a fast particle on the electron shells of the periodically located target atoms, and diffraction of the free photons arising at the input of a particle in the sample (i.e., diffracted transition radiation (DTR)) or those "born" in it due to the bremsstrahlung radiation (i.e., diffracted bremsstrahlung radiation (DBR). To estimate the characteristic mosaic angle $\sigma_{\mathrm{m}}$ of samples from the characteristics of the observed radiation is not of particular interest since the same information can be obtained by means of simpler X-ray analysis methods. However, analyzing the microstructure of samples, i.e., to estimate the dimensions of microblocks and disorientation angles of the neighboring blocks with respect to each other, is not obvious. When using electrons, by varying the observation angle and hence the energy of photons, it is possible to obtain information of a better quality than that when using the radiation with the fixed wavelength. Direct measurement of the dimensions of microblocks by means of $\mathrm{X}$-ray radiation beams is a complex experimental problem, and it can be used only to analyze the surface layers [4]. Therefore, to estimate the possibility of analyzing the microstructure of samples from the characteristics of the radiation caused by fast electrons passing through them is an important and topical problem.

\section{PROBLEM STATEMENT}

It is known that the characteristic width of the PXR angular distribution with respect to the Bragg order direction $\Theta_{\mathrm{ph}}=\sqrt{\gamma^{-2}+\omega_{\mathrm{p}}^{2} / \omega^{2}}$, where $\gamma$ is the Lorentz factor and $\omega_{\mathrm{p}}$ is the plasma frequency of the medium. As shown in [5], the total PXR intensity practically does not depend on the mosaic structure of a crystal. The mosaic structure leads to broadening of the PXR orientation dependence and angular distribution, and when $\Theta_{\mathrm{ph}}$ is comparable with the characteristic mosaic angle, it reduces the radiation yield due to the rereflection of the PXR photons [6, 7]. Therefore, the PXR sensitivity to the fine details of the crystal structure is 
insignificant. However, since for crystals with a perfect structure the PXR yield is much higher than the DBR yield [8], it is necessary to take into account the contribution of this mechanism to the observed radiation characteristics.

Diffracted photons are radiated in the Bragg direction, and the width of the orientation dependence is determined by the angular distribution of the primary radiation and, mainly, by the $\sigma_{\mathrm{m}}$ value and the collimation angle of the radiation $\vartheta_{c}$ [8]. In experiment, this value is rarely lower than several milliradians; therefore, by measuring the orientation dependence or angular distribution of the diffracted radiation (DBR or DTR) yield, one can estimate the mosaic structure of a crystal only for high enough $\sigma_{\mathrm{m}}$ values comparable with $\vartheta_{\mathrm{c}}$. Therefore, determining the mosaic structure and estimate the $\sigma_{\mathrm{m}}$ value is much easier and cheaper by means of the conventional X-ray diffraction methods.

The problem of whether it is possible to estimate the dimensions of the mosaic blocks and the characteristic angles of their mutual disorientation by measuring the spectral-angular radiation characteristics of fast electrons (PXR + DBR + DTR) in the sample is more important and interesting. Let the radiation with the spectral-angular distribution $I(\omega, \mathbf{n})$ be born on the surface or inside of the mosaic sample (respectively, transition and bremsstrahlung radiations). When the diffraction conditions hold on any plane of a separate mosaic block for photons with the energy $\omega$ and the unit vector in the direction of the motion of the photon $\mathbf{n}$ satisfying the condition

$$
\omega=\frac{|\mathbf{g}|^{2}}{2 \sqrt{\varepsilon_{0}}|\mathbf{n g}|}=\frac{g}{2 \sin \Theta_{\mathrm{B}}},
$$

where $g$ and $\Theta_{B}$ are the vector of the inverse lattice of this plane and the angle between it and the vector $\mathbf{n}, \mathbf{a}$ detector with the angular aperture $\vartheta_{c}$ located at the angle $\Theta_{D}=2 \Theta_{B}$ records the diffracted radiation. The intensity of this radiation depends on the reflecting ability of the sample.

It is known [9] that block crystals can be classified by the dimensions of the regular blocks or regions in the crystal and the degree of their mutual disorientation. According to the first issue, crystals can be divided into two types: $a$ and $b$. In $a$-type crystals, separate regions are large enough for the considerable influence of the primary extinction to be manifested, i.e., their linear dimension is comparable with the length of the primary extinction. In $b$-type crystals, the dimensions of the regular blocks are small; therefore, the effect of primary extinction is practically not observed. According to the second issue, the crystals can be also divided into two groups: $\alpha$ and $\beta$. In $\alpha$-group crystals, the blocks are almost parallel to each other and their mutual disorientation is small; therefore, the contribution of secondary extinction is high. In $\beta$-group crystals, the blocks are distributed irregularly; therefore, the contribution of secondary extinction is small.
By combining these issues, it is possible to conditionally divide the crystals into four groups from $a \alpha$ to $b \beta$ by the degree of perfection. The limit of the $a \alpha$ group, when the possible disorientation of the microblocks is lower than the region of the total reflection of X-rays (Darwin table), is an ideal perfect crystal, and that of the $b \beta$ group is an ideal mosaic crystal. Belonging to these groups is not fixed once and for all, since the extinction length, being a classification criterion, depends on the reflection order and energy of photons. That is, for different reflection orders or energy values of photons, the same sample can belong to different groups [9].

It is known [9] that the ability of the crystals to reflect X-ray radiation is related to the perfection of their structure. The crystals of the $a \alpha$ group provide the narrowest rocking curve (FWHM $\sim 20-30$ angl.s and less), described well by the dynamic theory of the $\mathrm{X}$-ray diffraction in perfect crystals. Therefore, their integral reflecting ability is low. $b$-type crystals have a much higher reflecting ability. The mosaic crystals of the $b \beta$ group have the maximum reflecting ability and the widest rocking curve. The reflecting ability of the mosaic $b$-type crystals is described well by the theory of X-ray diffraction in mosaic crystals. For crystals of the $b \alpha$ group, one has to additionally consider the secondary extinction, reducing the integral reflecting ability.

Crystals of the $a \alpha$ group can be sometimes presented in the form of a set of crystallites, i.e., smallsize perfect crystals, each of which reflects X-ray radiation according to the dynamic theory of diffraction. The reflecting ability of such a crystal as a whole is close to the reflecting ability of a perfect crystal with the same dimensions if the dimensions of the crystallite are larger than the length of the radiation absorption. Otherwise, the reflecting ability of such a crystal is higher than that of the perfect one since the radiation can be reflected from the crystallites located deeper along the motion of the beam [9]. It is necessary to emphasize that only in the ideal perfect and ideal mosaic crystals is the integral reflecting ability described well by the corresponding theories for any values of the energy of photons and reflection orders. In other cases, it is necessary to consider the dimensions of blocks and their distribution on the disorientation angles, which is, as a rule, not known.

When using crystals to generate the beams of the $\mathrm{X}$-ray radiation, a divergent photon beam with a continuous spectrum is incident on or born in the crystal, then a part of its photons is reflected and recorded on a detector with the fixed angular arrangement. In such a problem statement, the structure imperfection leads to the broadening of the spectrum of the detected radiation and the increase in its intensity. That is, for a crystal of the $a \alpha$ group, the recorded radiation has the lowest intensity and the width of the radiation spectrum and orientation dependence curve is mainly determined by the collimation angle of the radiation. 
For a crystal of the $b \beta$ group, the radiation intensity is maximum, as are the width of the radiation spectrum and orientation dependence curve. The dependence of the characteristics of the recorded radiation on the quality of the crystal structure allows one, in principle, to estimate the structure perfection of the real crystals by the characteristics of the observed radiation.

The characteristic parameters of the above classification of crystals, the width of the Darwin table $\Delta \Theta$ and the length of the primary extinction $l_{\mathrm{ex}}$, depend on the reflection order and energy of photons [10]. For nonpolarized radiation and no absorption, one can write

$$
\Delta \Theta=2 \gamma \Delta \theta_{0},
$$

where $\Delta \theta_{0}=2 \delta / \sin 2 \Theta$ is the correction to the Bragg angle due to the refraction of a wave in the crystal and $\delta=\left(\omega_{p} / \omega\right)^{2} / 2$ is the difference of the refraction parameter from unity,

$$
\gamma=\frac{1}{2} \frac{f(\mathbf{g})}{f(0)}(1+\cos (2 \Theta))
$$

Here $f(\mathbf{g})$ is the Fourier component of the spatial distribution of electrons in the crystal atom, a function of the atomic dispersion $(f(0)=z$, where $z$ is the number of electrons in the atom), and $\mathbf{g}$ is the vector of the inverse lattice corresponding to the plane where the reflection occurs.

As an estimate of the length of the primary extinction, one can use the expression [9]

$$
l_{\text {ex }}=d /(2 \bar{\xi} \sin \Theta),
$$

where $\exp (-2 \bar{\xi})$ is the weakening of the wave intensity when passing through one plane,

$$
2 \bar{\xi}=\frac{\pi d^{2} N F}{n} \frac{e^{2}}{m c^{2}}
$$

Here $N$ is the concentration of the dispersing centers, $d$ is the interplane distance, $F$ - is the structural coefficient, and $n$ is the reflection order.

To check the possibility of determining the imperfection degree of the crystal structure from the characteristics of the recorded radiation, it is desirable to analyze the results of the measurements for the same imperfect crystal and different energies of photons. In spite of a number of advantages of mosaic crystals when compared with perfect ones [7, 11], in the experiments on the PXR generation, as a rule, perfect crystals were used. The possible effect of structure imperfection, even if noted, was not commented on, and the measurements were performed for one observation angle. One can mention only the measurements performed on a Tomsk synchrotron for diamond targets cut out of the same sample of natural diamond for the observation angles $\Theta_{\mathrm{D}}=90^{\circ}[12]$ and $\Theta_{\mathrm{D}}=4^{\circ}[13]$. The results of processing the results of the measurements for each of the observation angles are published in $[6,11,14]$.

\section{EXPERIMENTAL DEVICE AND METHODS}

Measurements were performed on the internal electron beam of the Tomsk synchrotron. The experiment scheme is shown in Fig. 1a. The electrons accelerated to the final energy were thrown at a diamond target (DC) located on a goniometer. In the DC the radiation under study (PXR) was generated. The radiation spectrum and angular distribution were measured on a detector (D) located in the lead converter at the angle $\Theta_{D}$ with respect to the direction of the electron beam. The total energy of the bremsstrahlung radiation from the crystal was recorded on a Gauss quantometer $(\mathrm{Q})$.

In the Tomsk synchrotron, the electrons are focused on a target due to the losses on the synchrotron radiation [15]. After the desired energy of particles is achieved, the increase in the magnetic field stops and for $20 \mathrm{~ms}$ the field is kept constant with an accuracy not worse than $0.1 \%$. To increase the duration of the radiation pulse necessary for the normal work of the spectrometer equipment, the accelerating high-frequency (HF) field is not switched off, but smoothly decreases. Therefore the electrons leave synchronism with the accelerating field and are thrown at the target for 10-15 ms depending on the amplitude of the HF field. The spiralization step is about $0.01 \mathrm{~mm}$, and the maximal range of horizontal oscillations is from 1.5 to $2.5 \mathrm{~mm}$, depending on the operating mode of the accelerator and the energy of electrons. The distribution of electrons on the coordinate of hitting the target in the horizontal plane is described well by the distribution $f(x) \sim \exp (-x / \tau)$, where $x$ is the coordinate counted from the target edge closest to the equilibrium orbit, and $\tau$ is the characteristic parameter [16] (Fig. 1b). At the energy change from 900 to $500 \mathrm{MeV}$, the $\tau$ value decreases from $0.7-0.9$ [16] to $0.4 \pm$ $0.1 \mathrm{~mm}[17]$.

The distribution of electrons on the coordinates of hitting the target in the vertical planes (Fig. 1c) is described well by the Gaussian distribution $f(y)$ $\exp \left(-y^{2} /\left(2 \sigma^{2}\right)\right)$ with $\sigma \sim 0.6-0.7 \mathrm{~mm}[16]$ and is due to the vertical betatron oscillations [18]. The measurement of the beam sizes on the target [16] and in the equilibrium orbit [18] give the value of the divergence of the electron beam of the synchrotron $\vartheta_{\mathrm{e}} \sim 0.15-0.2 \mathrm{mrad}$.

The measurements were performed for two angles of the detector location: $\Theta_{\mathrm{D}}=90^{\circ}\left(\omega_{1} \sim 6.95 \mathrm{keV} \ll \gamma \omega_{\mathrm{p}}\right)$ [12] and $\Theta_{\mathrm{D}}=4^{\circ}\left(\omega_{1} \sim 148 \mathrm{keV}>\gamma \omega_{\mathrm{p}}\right)$ [13], where $\omega_{1}$ and $\omega_{\mathrm{p}}$ are the energy of photons for the first allowed reflection order and the energy of the medium plasmon, respectively. In both experiments, crystals cut out from the same sample of natural diamond [19] with dimensions of $6 \times 10 \times 0.35$ [12] and $6 \times 10 \times 2 \mathrm{~mm} \mathrm{[13]} \mathrm{and}$ surface mosaic structure $\sigma_{\mathrm{m}} \sim 0.2 \mathrm{mrad}[20]$ were used as targets. The $\langle 110\rangle$ direction was practically perpendicular to the large face, and the vertical and horizontal crystal faces were parallel to the (110) and (001) planes. 


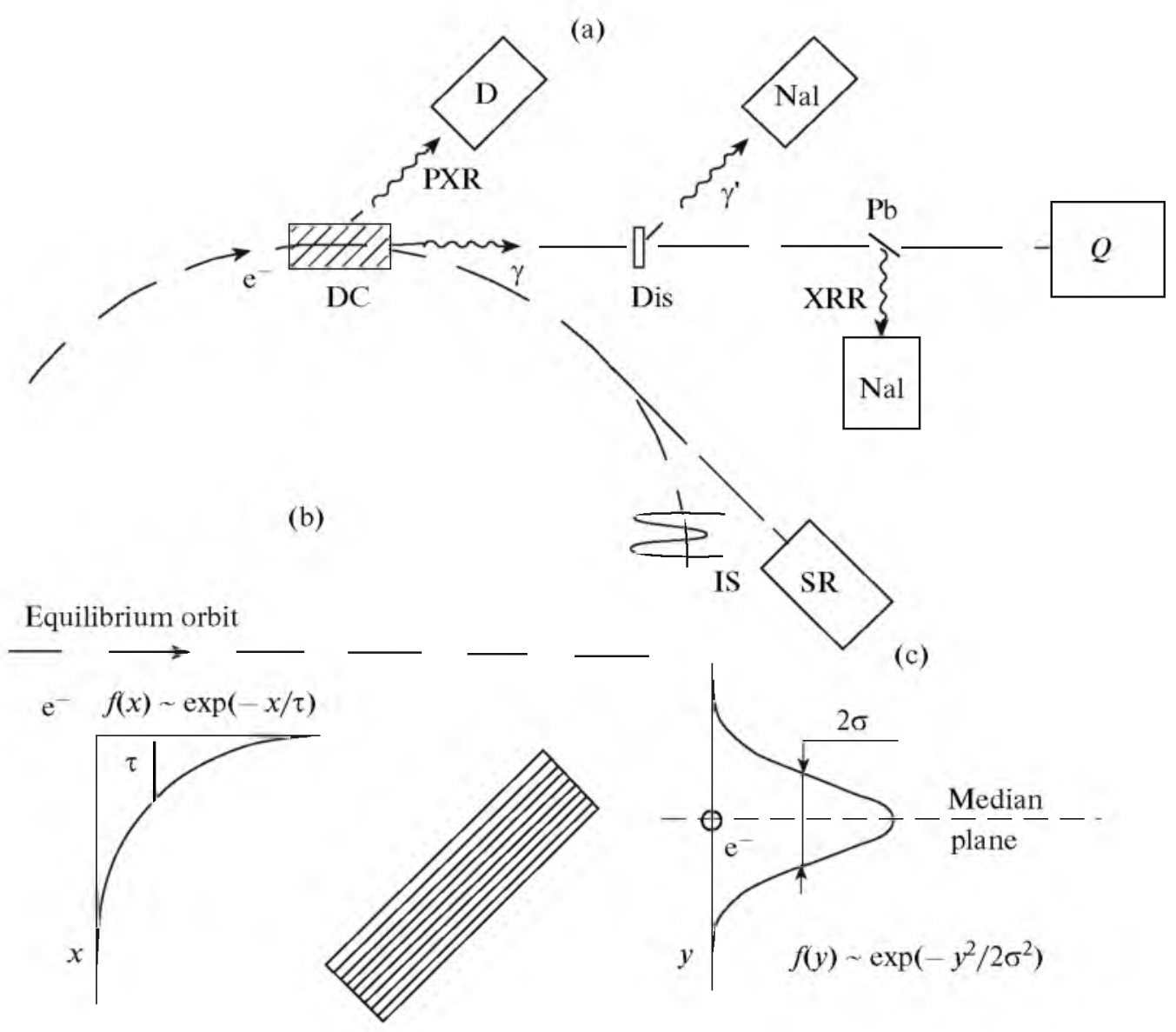

Fig. 1. Experimental scheme: Q — quantometer, DC—diamond crystal, D—radiation detector, IS — induction current sensor, $\mathrm{SR}$ - synchrotron radiation sensor, $\mathrm{Dis}$ - disperser, and $\mathrm{Pb}$ - lead converter.

In the spectral measurements, the current of the electron beam decreased to $\sim 10^{6}$ particles per pulse and was measured on a synchrotron radiation (SR) sensor. At such a current, for the time the electrons were thrown at the target $(\sim 10-15 \mathrm{~ms})$ the number of the detector response did not exceed 20-30 and the probability of overlapping of pulses from different photons did not exceed $3-5 \%$. The data of the synchrotron radiation sensor were absolutized by measuring the total energy of the bremsstrahlung radiation in the mode of the low (SR sensor) and high (induction sensor (IS)) currents for the same orientation of the target. The normalization error did not exceed $10-15 \%$ for the observation angle of $4^{\circ}$, the crystal thickness of $2 \mathrm{~mm}$, and the energy of electrons $E_{0}=500 \mathrm{MeV}$ [13]. In the experiment [12], for the observation angle of $90^{\circ}$ and $E_{0}=$ $900 \mathrm{MeV}$, the error increased because of the uncertainty of the average number of passages of electrons in the ring accelerator through a thin target and the strong dependence of this value on the crystal orientation [21] and was about $25-30 \%$. The accelerator parameters, the experimental scheme, and the technique of performing the measurements are described are described in detail in $[16,17,22]$.
For the observation angle $\Theta_{\mathrm{D}}=90^{\circ}$, the measurements were performed in the Bragg geometry. To record the 220 reflection, a crystal $0.35 \mathrm{~mm}$ thick was rotated by $45^{\circ}$ around the vertical axis and was oriented with the $(010)$ plane along the direction of the electron beam according to the indications of the $\mathrm{NaI}$ (T1) detector recording the radiation photons at channeling and bremsstrahlung radiation, dispersed in a converter (Dis) [23]. At such a location of the target, the electron beam was incident on the crystal at small angles to the $\langle 100\rangle$ direction and the vertical and horizontal crystal faces were parallel to the $\left(\begin{array}{lll}1 & 0\end{array}\right)$ and (001) planes.

Furthermore, the orientation was performed by the PXR yield recorded on a proportional counter with xenon filling BDP-2 with an aperture of $4 \times 16 \mathrm{~mm}$, efficiency $f \sim 80 \%$ for the energy of photons $\omega \sim 7 \mathrm{keV}$, and resolution $\Delta \omega / \omega \sim 15 \%$. To find the center of the PXR reflex and measure the angular distribution, a counter mounted at a distance of $106 \mathrm{~cm}$ from the crystal could be moved in vertical and horizontal directions with a step of $0.25 \mathrm{~mm}$. To measure the angular PXR distribution more accurately, a lead collimator with a diameter of $4 \mathrm{~mm}$ was mounted in front of the counter. 
The collimation angle of the radiation $\vartheta_{\mathrm{c}}=1.88 \mathrm{mrad}$ was smaller than the characteristic angular size of the beam of the PXR photons $\Theta_{\mathrm{ph}} \sim 6 \mathrm{mrad}$.

The angular PXR distribution was measured on a differential discriminator tuned to the energy of photons of $5.0<\omega<10.0 \mathrm{keV}$. When measuring the angular distribution of the background, a thin titan absorber $50 \mu \mathrm{m}$ thick was mounted on the path of the radiation beam from the target to the detector, which provided absorption of practically all PXR photons for the 220 reflection without changing other experimental conditions [14]. The angular PXR distribution for the first reflection order was obtained by subtracting the angular distributions measured without and with the absorber.

For the observation angle $\Theta_{D}=4^{\circ}$ and the energy of electrons of $500 \mathrm{MeV}$, the measurement was performed in the Laue geometry. The crystal $2 \mathrm{~mm}$ thick was mounted in the goniometer in such a manner that the (110) plane practically coincided with the vertical plane. The crystal was oriented with its plane along the direction of the electron beam by the radiation yield at the plane channeling by means of a thin NaI (TI) detector recording the change of the photon yield of the characteristic X-ray radiation from a lead converter $(\mathrm{Pb}) 0.4 \mathrm{~mm}$ thick and $5 \mathrm{~mm}$ wide mounted on the path of the beam of the $\gamma$-radiation from the diamond crystal with the orientation angle.

To achieve the 220 reflection, the crystal was rotated by the angle $\Theta \sim \Theta_{\mathrm{D}} / 2$. Further orientation was performed by the PXR yield recorded on a $\mathrm{NaI}(\mathrm{TI})$ detector with a diameter of $63 \mathrm{~mm}$ and thickness of $63 \mathrm{~mm}$ in front of which a collimator with a diameter of $14 \mathrm{~mm}$ was mounted. The detector was put at a distance of $364 \mathrm{~cm}$ from the crystal, which corresponded to the collimation angle of the radiation $\vartheta_{\mathrm{c}}=1.9 \mathrm{mrad}$. In the spectral range from 60 to $350 \mathrm{keV}$, the detector efficiency is close to unity. The resolution at the line was $59.4 \mathrm{keV}\left({ }^{241} \mathrm{Am}\right), \sigma=4.8 \pm 0.1 \mathrm{keV}$. The detector was calibrated and the linearity of the spectrometer path was checked by the $\gamma$-lines of the ${ }^{241} \mathrm{Am},{ }^{137} \mathrm{Cs}$ and ${ }^{22} \mathrm{Na}$ isotopes.

\section{ANALYSIS OF THE EXPERIMENTAL RESULTS}

For the experimental conditions [12], the energy of photons of the flrst reflection order $\omega_{1} \sim 6.95 \mathrm{keV} \ll$ $\gamma \omega_{p} \sim 67 \mathrm{keV}$; therefore, transition radiation is the main source of the radiation that can reach the detector after diffraction. The characteristic width of the angular PXR distribution $\Theta_{\mathrm{ph}} \sim 6 \mathrm{mrad}$ is much more than the characteristic radiation angle $\gamma^{-1} \sim 0.6 \mathrm{mrad}$; therefore, the measurement of the angular distribution of the resultant radiation allows the separation of the DTR and PXR contributions.

Figure 2 shows the results of measurement of the vertical angular distribution of the radiation yield for the 220 reflection in the experiment [12]. The dependence

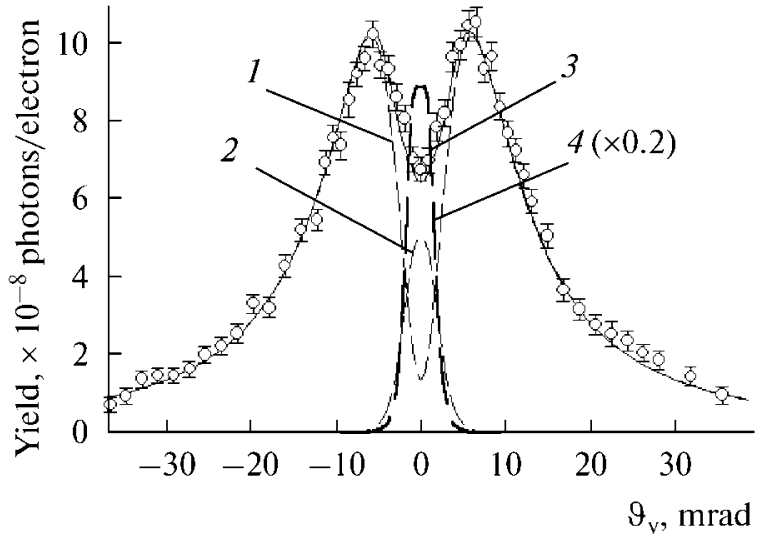

Fig. 2. Vertical angular distribution of the photons of the first reflection order for the experiment in [12]: $E_{0}=$ $900 \mathrm{MeV}, T=0.5 \mathrm{~mm}, \Theta_{\mathrm{D}}=90^{\circ}$, and $\vartheta_{\mathrm{C}}=1.88 \mathrm{mrad}$. Points, experiment. Curves, calculation: $l-P X R$ in the perfect crystal, 2-DTR in the perfect crystal, 3-PXR + DTR in the perfect crystal, and 4-DTR in the mosaic crystal of the group $b \alpha$.

1 gives the results of the calculation according to the kinematic PXR theory [24] assuming that the crystal is perfect [14] and the electrons pass through the target only once. As shown in $[6,11]$, when the $\sigma_{\mathrm{m}} \ll \Theta_{\mathrm{ph}}$ condition holds as in this case, the mosaic structure practically does not affect the spectrum and the angular PXR distribution. Similarly to [14], due to the absolutization error $(-20-30 \%)$ and the uncertainty of the average number of passages of electrons through the thin target, the results of the measurements are normalized to the calculation results of in the range of the angles of the start of photons $\vartheta_{V} \geq 5 \mathrm{mrad}$. One can see in the figure that the calculated dependence describes well the experimental results only for angles $\vartheta_{V} \geq 5-7 \mathrm{mrad}$. For smaller angles, the experimental points are much higher than the calculated curve indicating the contribution of the radiation with a narrower angular distribution than that of PXR, i.e., the DTR contribution.

Figure 2 (curve 2) shows the results of the calculation of the vertical dependence of the DTR yield performed according to [25] for the perfect crystal and the single passage of electrons through the target. In the calculation, the geometrical dimensions of the detector and the cross-sectional dimensions of the electron beam (Fig. 1b, c) are taken into account. For the conditions of the experiment under study, the dimensions of the detector are larger than the dimensions of the electron beam of the target and the collimation angle $\vartheta_{\mathrm{c}}=1.88 \mathrm{mrad}$ is somewhat larger than $\gamma^{-1}=0.6 \mathrm{mrad}$; therefore, the angular DTR distribution is practically not affected by taking into account geometrical factors. When the dimensions of the detector and the energy of electrons are decreased, as, for example, in the experiment in [25], the angular distributions calculated with and without taking into account the geo- 


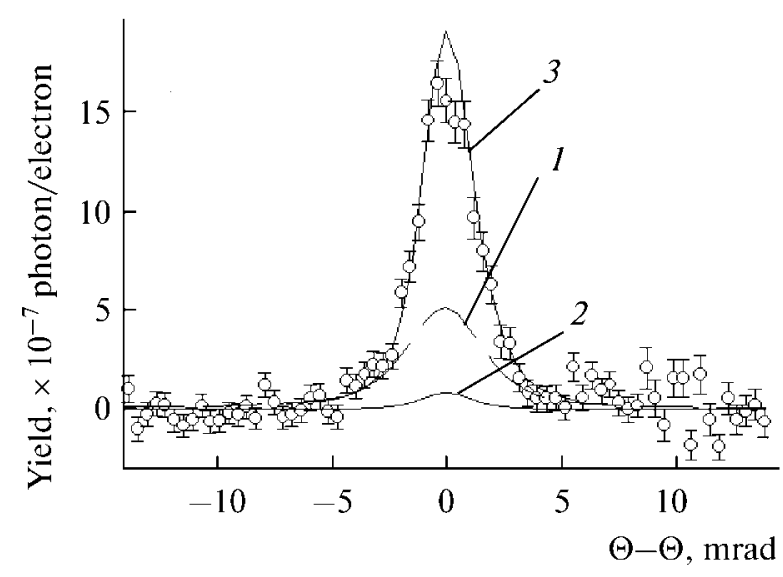

Fig. 3. Orientation dependence of the yield of photons of the first reflection order for the experiment in [13]: $E_{0}=$ $500 \mathrm{MeV}, T=2 \mathrm{~mm}, \Theta_{\mathrm{D}}=4^{\circ}$, and $\vartheta_{\mathrm{c}}=1.9 \mathrm{mrad}$. Points, experiment. Curves, calculation: $1-\mathrm{PXR}$ in the perfect crystal, 2-DBR in the perfect crystal, and 3-PXR + DBR in the mosaic crystal of the group $b \alpha$.

metrical dimensions of the detector and the electron beam differ considerably. To obtain agreement of the experimental and calculated dependences, the authors of [25] used the divergence of the electron beam of the Tomsk synchrotron as a free parameter and obtained the value $\vartheta_{\mathrm{e}}=1 \mathrm{mrad}$ instead of $\vartheta_{\mathrm{e}} \sim 0.2 \mathrm{mrad}$ [16].

Curve 3 in Fig. 2 shows the resultant angular PXR + DTR distribution. One can see from the figure that the calculated dependence coincides well with the experimental one. In the center of the angular distribution, the experimental and calculated dependences somewhat differ, which may be due to both the uncertainty of some parameters used in the calculation (the dimensions of the electron beam and its divergence) and the mosaic structure of the crystal. The calculations show that the increase in the divergence of the beam due to the difference of average ratio of passages of electrons through the crystal from unity [21] practically does not affect the form of the angular distribution of detected radiation.

The characteristic angle of the mosaic structure of this crystal $\sigma_{\mathrm{m}} \sim 0.2 \mathrm{mrad}[20]$ is comparable with the width of the region of the total reflection for this energy of photons $\Delta \Theta \sim 0.02 \mathrm{mrad}$. Therefore, according to the disorientation degree of the blocks, this crystal should be attributed to the $\alpha$ group. To check the applicability of the approximation of the mosaic $b$-type crystal to this sample, the angular DTR distribution was calculated in the framework of this approximation according to the technique described in $[17,26]$ (Fig. 2, curve 4). Figure 2 shows that the angular DTR distributions for the perfect crystal (curve 2) and the mosaic $b$-type crystal (curve 4) are similar but differ in intensity practically by an order of magnitude. Such a ratio should be approximately expected, since, for the mosaic $b$-type crystals, the reflecting ability $Q \sim N^{2} \lambda^{2}$
$[6,10,27]$, where $N$ is the concentration of the dispersing centers and $\lambda$ is the wavelength. Thus, for relatively large wavelengths, the probability of the reflection of the X-ray radiation by a sample of such thickness, just as for a perfect crystal, is close to unity and the characteristic angle of the mosaic structure is approximately an order of magnitude larger than the width of the Darwin table.

Dependence 3 is considerably closer to the experimental points than the sum of curves 1 and 4 (Fig. 2); therefore, the diamond crystal used in [12] should be attributed the the $a \alpha$ group; i.e., the dimensions of the surface mosaic stricture in the experiment [20] are larger than the length of the primary extinction $l_{\mathrm{ex}} \sim 7 \mu \mathrm{m}$.

With an increase in the energy of photons, the length of the primary extinction increases and the situation changes. The points in Fig. 3 show the dependence of the yield of photons of the first reflection order on the disorientation angle of the (110) plane of the diamond crystal $2 \mathrm{~mm}$ thick with respect to the direction of the Bragg reflection obtained in the experiment in [13] after subtracting the background, the level of which did not exceed $30 \%$ of the yield in the maximum. The errors are statistical and do not include the error of determining the number of electrons passing through the crystal. The results of this experiment were already discussed in $[6,11]$.

Here (Fig. 3, curve 1) the calculated dependence obtained in the framework of the kinematic PXR theory is shown. The major factors determining the form of the orientation dependence (OD) of the radiation yield are the collimation angle of the radiation and the multiple dispersion of particles in the crystal. Therefore, the measured and calculated ODs are close enough in form, but considerably differ in amplitude. The difference in the half-width (FWHM) of the calculated and measured dependences $\Delta \Theta_{\text {calc }}=3.97 \mathrm{mrad}$ and $\Delta \Theta_{\text {exp }}=(2.9 \pm 0.2) \mathrm{mrad}$ exceeds the experimental error (the step of measuring $\mathrm{OD} \approx 0.4 \mathrm{mrad}$ ) and also indicates contribution of radiation with a narrower angular distribution than that of the PXR. For the experimental conditions of the cited reference $\omega \approx 145 \mathrm{keV}>\gamma \omega_{\mathrm{p}} \sim 35 \mathrm{keV}$, this is the diffracted bremsstrahlung radiation.

The estimation of the DBR contribution according to the technique of [9] for a perfect crystal (Fig. 3, curve 2) has shown that, in this case, the DBR intensity does not exceed $25 \%$ of the PXR yield and cannot explain the experimental results. Taking into account the mosaic structure of the crystal according to the technique of $[6,17,26]$ in the case of homogeneous distribution of mosaic blocks with dimensions smaller than the length of the primary extinction $l_{\mathrm{ex}}=148 \mu \mathrm{m}$ (mosaic $b$-type crystals) has shown that the observed difference is in fact due to the mosaic structure of the crystal. The resultant (AT + DBR) dependence calculated by taking into account the mosaic structure for 
both components (Fig. 3, curve 3) is close to the experimental one. The calculated values of the yield $Y^{\text {calc }}=1.94 \times 10^{-6}$ photons/electron and the $\mathrm{OD}$ width $\Delta \Theta_{\text {calc }}=2.67 \mathrm{mrad}$ agree well with the results of the measurements $Y^{\text {exp }}=(1.63 \pm 0.008) \times 10^{-6}$ photons/electron.

The difference between the calculation and experimental results may be due to the normalization error, assumption of the homogeneity of the distribution of the blocks on the crystal thickness, and the used estimation of the $\sigma_{\mathrm{m}}$ value. Depending on the $\sigma_{\mathrm{m}}$ value and the ratio of the "mosaic" to the "perfect" components of the crystal the radiation yield and OD width change. Another origin of this difference can be recording several photons from different electrons as a single photon of higher energy (overlapping effect). The possibility of this effect is indicated by the fact that the position of the maximum of the orientation dependence does not coincide with its center.

As it was already noted, both crystal targets are cut out from the same sample of the natural diamond; therefore, their microstructure should be approximately identical. This is confirmed by the results of the experiment of [28], in which the radiation spectra of these crystals for the observation angle $\Theta_{D}=90^{\circ}$ and $E_{0}=900 \mathrm{MeV}$ were recorded on the detector with the collimation angle $\vartheta_{\mathrm{c}}=25 \mathrm{mrad} \gg \Theta_{\mathrm{ph}}$. If the microstructure of the crystals differs and for the energy of photons $\omega_{1} \sim 6.8 \mathrm{keV}$, the crystal $2 \mathrm{~mm}$ thick belongs to the $b \alpha$ group for which the high yield of the DTR photons (Fig. 2, curve 4) should be recorded and that $0.35 \mathrm{~mm}$ thick belongs to the $a \alpha$ group where the yield of the DTR photons is rather small; the orientation dependences of the radiation yield for these crystals should differ, but this fact is not noted by the authors of the cited reference.

In the experiment of [17], the crystals of pyrolitic graphite with characteristic dimensions of mosaic blocks $1 \sim 1-5 \mu \mathrm{m}$ [4] for the same observation angles corresponded to the $b \alpha$ group, that is, the main contribution to the radiation yield was provided by the DTR and DBR mechanisms for the observation angles of $90^{\circ}$ and $4^{\circ}$, respectively. Therefore, one can assume that the results of the measurements for the observation angle of $90^{\circ}$ [12] give the lower estimate of the characteristic dimensions of the blocks $l>l_{e x} \approx 7 \mu \mathrm{m}$ and the results for the observation angle of $4^{\circ}[13]$ give the upper estimate of this value $l \ll l_{e x} \approx 148 \mu \mathrm{m}$. Hence, the characteristic dimensions of the microblocks in the sample of the natural diamond from which the targets used in the experiments in [12] and [13] were cut out are within the limits of $7 \mu \mathrm{m}<l<148 \mu \mathrm{m}$.

With an increase in the energy of photons for any imperfect crystal, the applicability of the model of the mosaic $b$-type crystal becomes more justified [10]. Experience shows that the PXR theory in the kinematic approximation describes the results of the mea- surements in perfect crystals with the accuracy not worse than $10-15 \%$ [29]. With about the same accuracy, the theory of the diffraction of the X-ray radiation in mosaic crystals describes the yield of the diffracted real photons in the $b$-type crystals [22]. Hence, one can determine at what observation angle the transition from one group to another begins, i.e., estimate the average dimension of the blocks composing the investigated sample from the results of the measurements of the orientation dependences or angular distributions of the radiation yield for several observation angles and the comparison with the results of the calculation of the yield of the PXR and diffracted real photons (DBR or DTR) for mosaic and perfect crystals. The same measurements can be used to choose the crystals for obtaining intense $\mathrm{X}$-ray radiation beams at the passage of fast electrons through the mosaic crystals of the $b \alpha$ group for various, including medical, applications [7, 11].

The measurements performed for several observation angles are time-consuming both due to the time needed to perform the measurements and to reinstall the radiation detectors and to choose the optimum operating modes of the accelerator and the experimental equipment. According to Eqs. (1)-(3), an analogous change of the width of the Darwin table and the length of the primary extinction, the same as when using different observation angles, occurs with an increase in the reflection order due to the reduction of the $F$ value and the $n^{-1}$ factor. Therefore, the information on the dimensions of blocks can be obtained faster during the measurements of the angular distributions or orientation dependence of the radiation yield for a single observation angle but several reflection orders. Usually, in an experiment one records reliably from three to six or seven reflection orders depending on the type of the crystal under study and the data-acquisition equipment used $[14,30]$, which is analogous to using one reflection order and several observation angles.

\section{CONCLUSIONS}

The following conclusions were drawn from the experimental results:

(1) The dependence of the radiation intensity at the passage of fast electrons in the crystal on the imperfection of its structure allows one to state the problem of the quantitative estimation of the defects of the structure of crystal samples from the characteristics of the recorded radiation.

(2) The weak dependence of the PXR intensity on the mosaic structure of the crystal does not allow one to use effectively this type of radiation for the analysis of the microstructure.

(3) One can obtain information about the characteristic dimensions of the blocks forming the sample under investigation when the dimensions of the blocks are of the order of the length of the primary extinction 
by recording the radiation yield at the Bragg angles (DTR + PXR + DBR) for different observation angles (different reflection orders) and comparing these data with the calculation results for these experimental conditions.

\section{ACKNOWLEDGMENTS}

We are grateful to the coauthors of $[12,13,17,26]$ for their participation in the development and implementation of the techniques used in the research and help in the measurements. The study is supported by the Program of Internal Grants of Belgorod State University.

\section{REFERENCES}

1. V. A. Ryabov, Channalling Effect (Energoatomizdat, Moscow, 1994) [in Russian].

2. V. G. Baryshevskii and I. D. Feranchuk, Zh. Eksp. Teor. Fiz. 61, 944 (1971) [Sov. Phys. JETP 34, 502 (1971)].

3. G. M. Garibyan and Yan Shi, Zh. Eksp. Teor. Fiz. 61, 930 (1971) [Sov. Phys. JETP 34, 495 (1971)].

4. M. Ohler, J. Baruchel, A. W. Moore, Ph. Galez, and A. Freund, Nucl. Instrum. Methods Phys. Res. B 129, 257 (1997).

5. A. M. Afanas'ev and M. A. Aginyan, Zh. Eksp. Teor. Fiz. 74, 570 (1978) [Sov. Phys. JETP 47, 300 (1978)].

6. A. N. Baldin, I. E. Vnukov, D. A. Nechaenko, and R. A. Shatokhin, Vestn. KhNU, Ser. Fiz. 744 (3), 51 (2006).

7. I. E. Vnukov, Yu. V. Zhandarmov, and R. A. Shatokhin, Vestn. KhNU, Ser. Fiz. 808 (2), 25 (2008).

8. K.-H. Brenzinger, C. Herberg, B. Limburg, et al., Z. Phys. A 358, 107 (1997).

9. D. A. Baklanov, A. N. Baldin, I. E. Vnukov, et al., Vestn. KhNU. Ser. Fiz. 763 (1), 41 (2007).

10. R. James, Optical Principles of the Diffraction of X-rays (Cornell Univ., Ithaca, 1965; Inostr. Liter., Moscow, 1950).

11. A. N. Baldin, I. E. Vnukov, and R. A. Shatokhin, Pis'ma Zh. Tekh. Fiz. 33 (14), 87 (2007) [Tech. Phys. Lett. 33, $625(2007)]$.
12. V. A. Verzilov, I. E. Vnukov, V. V. Zarubin, et al., in Proc. of the 27th Intern. Conf on Physics of Interaction of Charged Particles with Crystals (Mosc. Gos. Univ., Moscow, 1997), p. 72.

13. O. V. Chefonov, B. N. Kalinin, G. A. Naumenko, et al., in Proc. of the 4th Intern. Symp. on Radiation from Relativistic Electrons in Periodic Structures, Sept., 13-16, 1999, Lake Baikal, Russia.

14. A. N. Baldin, I. E. Vnukov, B. N. Kalinin, and E. A. Karataeva, Poverkhnost', No. 4, 72 (2006).

15. L. D. Butakov, E. G. Gal', G. F. Kiryukhina, et al,, Izv. Vyssh. Uchebn. Zaved., Ser. Fiz. 34 (6), 5 (1991).

16. Yu. N. Adishchev, S. A. Vorob'ev, V. N. Zabaev, et al,, Yad. Fiz. 35, 108 (1982) [Sov. J. Nucl. Phys. 35, 63 (1982)].

17. I. E. Vnukov, B. N. Kalinin, G. A. Naumenko, et al., Izv. Vyssh. Uchebn. Zaved., Ser. Fiz. 44 (3), 53 (2001).

18. A. V. Kozhevnikov, M. M. Nikitin, and A. F. Medvedev, Izv. Vyssh. Uchebn. Zaved., Ser. Fiz., No. 10, 115 (1971).

19. A. P. Potylitsyn, Izv. TPU 301, 55 (2000).

20. E. I. Rozum, Dep. VINITI from 03.10.79, No. 3459 (Red. Zh. Izv. Fiz., Tomsk, 1979).

21. B. N. Kalinin, A. A. Kurkov, and A. P. Potylitsyn, Izv. Vyssh. Uchebn. Zaved., Ser. Fiz. 34 (6), 81 (1991).

22. E. A. Bogomazova, B. N. Kalinin, G. A. Naumenko, et al., Nucl. Instrum. Methods. Phys. Res B 201, 276 (2003).

23. B. N. Kalinin, E. I. Konovalova, G. A. Pleshkov, et al, Prib. Tekh. Eksp., No. 3, 31 (1985).

24. H. Nitta, Phys. Lett. A 158, 270 (1991).

25. Y. N. Adischev, S. N. Arishev, I. E. Vnukov, et al., Nucl. Instrum. Methods. Phys. Res B 201, 114 (2003).

26. I. E. Vnukov, B. N. Kalinin, A. A. Kir'yakov, et al., Izv. Vyssh. Uchebn. Zaved., Ser. Fiz. 44 (3), 71 (2001).

27. M. Chabot, P. Nicolai, K. Wohrer, et al., Nucl. Instrum. Methods. Phys, Res. B 61, 377 (1991).

28. Yu. N. Adishchev, S. A. Vorob'ev, B. N. Kalinin, et al., Zh. Eksp. Teor. Fiz. 90, 829 (1986) [Sov. Phys. JETP 63, 484 (1986)].

29. K.-H. Brenzinger, B. Limburg, H. Backe, et al., Phys. Rev. Lett. 79, 2462 (1997).

30. R. B. Fiorito, D. W. Rule, X. K. Maruyama, et al., Phys. Rev. Lett. 71, 704 (1993). 